The current findings raise questions about the evolutionary conservation of the mechanisms by which $\mathrm{PcG}$ proteins mediate gene silencing. Although the PcG proteins are evolutionarily conserved, there may be differences among their mechanisms. In Drosophila, $\mathrm{PcG}$ proteins are recruited to their target loci by specific regulatory sequences termed Polycomb response elements (PRE). No PRE has been identified in mammals ${ }^{3}$. In Drosophila, the products of gap and pair-rule genes target large complexes of

PcG proteins to Hox loci in the early embryo, but there is no evidence of a similar mechanism in mammals. In addition, the new findings imply that in mammals, gene silencing by PcG proteins involves DNA methylation, but this is highly unlikely in Drosophila owing to the absence of Dnmt1 and Dnmt3 homologs or any appreciable DNA methylation ${ }^{18}$.

Finally, the findings by Viré et al. ${ }^{1}$ and Hernández-Muñoz et al. ${ }^{2}$ have potential implications for altered nuclear structure in

\section{Helicase à go-go-go}

Helicases use the energy derived from NTP hydrolysis to pry apart the hydrogen bonds between base pairs. These enzymes have been characterized in various ways, but most methods report only their bulk macroscopic properties. The ability to follow helicase activity at the single-molecule level can provide insights into how the hydrolysis of cancer. Cancer-cell genomes often show global hypomethylation with hypermethylation at specific loci. Hypermethylation of the promoter regions of tumor-suppressor genes correlates with transcriptional repression, but it is not known which change occurs first ${ }^{19}$. It is possible that $\mathrm{PcG}$ recruitment of DNMTs could result in the observed hypermethylation. Gene repression at hypermethylated sequences could be mediated by PRC1 components, possibly including BMI1, and the loss of repressive states in hypomethylated regions could be due to the failure to recruit PRC1 components. Before these issues can be addressed, we will need a clear understanding of the functional significance of the interactions between PcG proteins and DNA methyltransferases.

1. Viré, E. et al. Nature, advance online publication 14 December 2005 (doi:10.1038/nature04431).

2. Hernández-Muñoz, I., Taghavi, P., Kuijl, C., Neefjes, J. \& van Lohuizen, M. Mol. Cell. Biol. 25, 11047-11058
(2005).

3. Ringrose, L. \& Paro, R. Annu. Rev. Genet. 38, 413-443 (2004).

4. Bestor, T.H. \& Verdine, G.L. Curr. Opin. Cell Biol. 6, 380-389 (1994).

5. Bestor, T., Laudano, A., Mattaliano, R. \& Ingram, V. J. Mol. Biol. 203, 971-983 (1988).

6. Chen, T., Ueda, Y., Dodge, J.E., Wang, Z. \& Li, E. Mol. Cell. Biol. 23, 5594-5605 (2003).

7. Rhee, I. et al. Nature 416, 552-556 (2002).

8. Okano, M., Bell, D.W., Haber, D.A. \& Li, E. Cell 99, 247-257 (1999).

9. Xu, G.L. et al. Nature 402, 187-191 (1999).

10. Ioshikhes, I.P. \& Zhang, M.Q. Nat. Genet. 26, 61-63 (2000).

11. Kirmizis, A. et al. Genes Dev. 18, 1592-1605 (2004).

12. Rollins, R.A. et al. Genome Res. published online 19 December 2005 (doi:10.1101/gr.4362006).

13. Martens, J.H. et al. EMBO J. 24, 800-812 (2005).

14. Lubit, B.W., Pham, T.D., Miller, O.J. \& Erlanger, B.F. Cell 9, 503-509 (1976).

15. Saurin, A.J. et al. J. Cell Biol. 142, 887-898 (1998).

16. Bestor, T.H. Trends Genet. 19, 185-190 (2003).

17. Mager, J., Montgomery, N.D., de Villena, F.P. \& Magnuson, T. Nat. Genet. 33, 502-507 (2003).

18. Goll, M.G. \& Bestor, T.H. Annu. Rev. Biochem. 74 481-514 (2005).

19. Baylin, S. \& Bestor, T.H. Cancer Cell 1, 299-305 (2002). NTP is directly coupled to translocation, unwinding of the duplex and processivity.

In a recent study, Dumont et al. (Nature 439, 105-108, 2006) use a single-molecule apparatus with high spatial and temporal resolution to examine the activities of hepatitis C virus NS3, an RNA helicase. Monomeric NS3 and ATP were placed in a chamber in which a 60-base-pair (bp) RNA hairpin with long single-strand arms was held by optical tweezers. Initially, NS3 binds

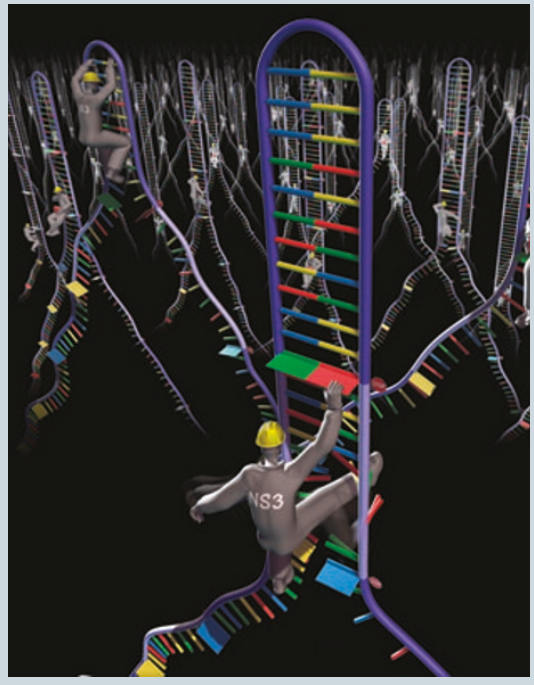

the single-stranded region of the hairpin and translocates in the $3^{\prime} \rightarrow 5^{\prime}$ direction. When NS3 encounters the duplex region and begins unwinding, the length of the RNA (reflected in the distance between the beads at the ends of the RNA) increases. Once the enzyme completes unwinding and dissociates, the hairpin re-forms, enabling multiple measurements from a single RNA molecule. In the illustration, NS3 is depicted as a workman climbing up a base-paired ladder, disrupting the steps as he moves.

Surprisingly, the unwinding traces are not monotonic but alternate between periods of rapid strand separation and pauses. This behavior is nonrandom; the rapid unwinding corresponds to a distance of $11 \pm 3 \mathrm{bp}$, which is consistent with the enzyme's step size as obtained from previous bulk measurements (the pause is shown as a stop sign every 11 steps). In rare instances, backward movement over the same with its translocation.

distance was observed. As individual unwinding events were recorded, information about the steps involved in unwinding could be extracted. For example, exit from a pause requires ATP binding, as expected, but it also has an ATPindependent component, the nature of which remains to be determined. The rate at which unwinding occurs between pauses (with $v_{\max }=$ $51 \mathrm{bp} \mathrm{s}^{-1}$ ) is dependent on ATP concentration. This result suggested that there are substeps within each 11-bp translocation, and careful examination of the tracings revealed the existence of 3.6-bp substeps, which has not been seen before. This observation is consistent with three cycles of ATP binding and hydrolysis per 11-bp unwinding event. Finally, processivity experiments indicated that NS3 is limited by its ability to translocate rather than its ability to separate strands and that reannealing competes

Overall, the kinetic properties determined by the single-molecule approach approximate the bulk measurements, with some of the differences attributed to the form (monomeric versus dimeric) of NS3 used. The authors propose a model for NS3 helicase activity, taking into account that there are two RNA-binding sites per monomer. In this model, one site is thought to contact the duplex $11 \mathrm{bp}$ ahead of the enzyme after the paused enzyme binds ATP. The other (lagging) RNA-binding site is thought to act in duplex opening, translocating forward $3.6 \mathrm{bp}$ during each of the three sequential ATP-binding and hydrolysis reactions. Other enzymes that disrupt base-pairing, such as polymerases, may also be studied using a variant of this assay. This may reveal some of the secrets of these molecular motors.

Angela K Eggleston 